INJE-TP-05-05, hep-th/0506096

\title{
No Hawking-Page phase transition in three dimensions
}

\author{
Y. S. Myung* \\ Relativity Research Center, Inje University, Gimhae 621-749, Korea \\ and Institute of Theoretical Science, University of Oregon, Eugene, OR 97403-5203, USA
}

\begin{abstract}
We investigate whether or not the Hawking-Page phase transition is possible to occur in three dimensions. Starting with the simplest class of LanczosLovelock action, thermodynamic behavior of all AdS-type black holes without charge falls into two classes: Schwarzschild-AdS black holes in even dimensions and Chern-Simons black holes in odd dimensions. The former class can provide the Hawking-Page transition between Schwarzschild-AdS black holes and thermal AdS space. On the other hand, the latter class is exceptional and thus the Hawking-Page transition is hard to occur. In three dimensions, a second-order phase transition might occur between the non-rotating BTZ black hole and the massless BTZ black hole (thermal AdS space), instead of the first-order Hawking-Page transition between the non-rotating BTZ black hole and thermal AdS space.
\end{abstract}

*Email-address : ysmyung@physics.inje.ac.kr 


\section{INTRODUCTION}

Hawking's semiclassical analysis for the black hole radiation suggests that most of information in initial states is shield behind the event horizon and is never back to the asymptotic region far from the evaporating black hole [1]. This means that the unitarity is violated by an evaporating black hole. However, this conclusion has been debated ever since [2-4]. It is closely related to the information loss paradox which states the question of whether the formation and subsequent evaporation of a black hole is unitary. One of the most urgent problems in the black hole physics is to resolve the unitarity issue.

Recently, Maldacena proposed that the unitarity can be restored if one takes into account the topological diversity of gravitational instantons with the same AdS boundary in threedimensional gravity [5]. Actually, three-dimensional gravity [6] is not directly related to the information loss problem because there is no physically propagating degrees of freedom [7]. If this gravity is part of string theory [8], the AdS/CFT correspondence [9] means that the black hole formation and evaporating process should be unitary because its boundary can be described by a unitary CFT. On later, Hawking has withdrawn his argument on information loss and suggested that the unitarity can be preserved by extending Maldacena's proposal to four-dimensional gravity system [10]. In other words, the topological diversity is credited with the restoration of S-matrix unitarity in the formation and evaporation of a black hole. In this approach the thermal AdS space plays an important role in restoring unitarity.

We remark an interesting phenomenon in the AdS black hole thermodynamics. There exists the Hawking-Page transition between AdS-Schwarzschild black hole and thermal AdS space in four dimensions [11]. This transition was based on the semi-classical approximation of the Euclidean path integral for the black hole thermodynamics. Some authors have proposed that this transition is possible in three-dimensional spacetimes: transition occurs between the non-rotating BTZ black hole and thermal AdS space [12]. Since the three-dimensional gravity and its boundary CFT can provide a prototype to compute bulk thermodynamic quantities and boundary thermal correlators exactly, they play an important role for investigating the evaporation of the black hole through the Hawking radiation.

In this letter we show that it is hard to occur the first-order Hawking-Page transition between the non-rotating BTZ and thermal AdS space. Instead, second-order transitions between the non-rotating BTZ black hole and the massless BTZ black hole (thermal AdS space) might be possible to occur in three dimensions.

For this purpose, we use the higher-order gravity theory in any dimension which may include quantum effects. According to a work of Ref. [13], all thermodynamic behaviors of AdS black holes were classified into two types: AdS-Schwarzschild black holes in even dimensions and Chern-Simons black holes in odd-dimensions. The first type possesses a continuous mass spectrum whose vacuum with zero mass is the thermal AdS space, whereas the second one has a continuous mass spectrum whose vacuum is the massless extremal AdS black hole.

In this work we consider three interesting cases [14-17]. 1) The non-rotating BTZ black hole (NBTZ) with $M>0, J=0: r_{+}=l \sqrt{M}, T_{H}=\frac{r_{+}}{2 \pi l^{2}}, C_{J}=4 \pi r_{+}=S_{B H}$. 2) The thermal AdS spacetime (TADS) with $M=-1, J=0$. We choose $T_{H}=0, C_{J}=0, S_{B H}=$ 0 because of the absence of the event horizon. This case corresponds to the spacetime picture of the NS-NS vacuum state [18]. 3) The massless BTZ black hole (MBTZ) with 
$M=J=0: T_{H}=0, C_{J}=0, S_{B H}=0$. This is called the spacetime picture of the RR vacuum state. Although the thermodynamic properties of TADS and MBTZ are nearly the same, their Euclidean topologies are quite different: TADS (MBTZ) are topologically trivial (non-trivial).

The organization of this letter is as follows. Section II is devoted to studying all AdS black holes in the simplest framework of Lanczos-Lovelock gravity. In section III, we show that there is no Hawking-Page transition in three dimensions.

\section{THERMODYNAMICS OF ADS BLACK HOLES}

We start with the simplest class of Lanczos-Lovelock theory in $d$-dimensional AdS spacetimes [13]

$$
I_{k}=\kappa \int \sum_{p=0}^{k} c_{p}^{k} \mathcal{L}^{(p)}
$$

with

$$
c_{p}^{k}=\frac{l^{2(p-k)}}{d-2 p}\left(\begin{array}{l}
k \\
p
\end{array}\right), p \leq k ; \quad c_{p}^{k}=0, p>k .
$$

Here an integer $k$ with $1 \leq k \leq[(d-1) / 2]$ represents the highest power of curvature in the lagrangian and a parameter $\kappa$ is related to the $d$-dimensional gravitational constant $G_{k}$ as $\kappa=1 / 2(d-2) ! \Omega_{d-2} G_{k}$. For a given $d, c_{p}^{k}$ describes a family of inequivalent theories labelled by $k . \mathcal{L}^{(0)}$ is given by the cosmological constant $\Lambda=-(d-1)(d-2) / 2 l^{2}$ with the AdS curvature radius $l$ and $\mathcal{L}^{(1)}$ is the curvature scalar $R$. $\mathcal{L}^{(2)}$ corresponds to the Gauss-Bonnet term of $-R_{\mu \nu \alpha \beta} R^{\mu \nu \alpha \beta}+4 R_{\mu \nu} R^{\mu \nu}-R^{2}$, whose equation of motion contains no more than second-order derivatives of the metric and which appears to be a ghost free theory. This term is relevant to the case with $d \geq 5$. Further, $I_{1}$ corresponds to the $d$-dimensional EinsteinHilbert action including the negative cosmological constant $\Lambda$. If $d=2 n-1(n \in \mathbf{N})$, the maximum value of $k$ is given by $k=n-1$. In this case the corresponding lagrangian is the Chern-Simons $(2 n-1)$-form. For $d=2 n$ and $k=n-1$, the action is defined by the 2 -form Pfaffian and it takes the Born-Infeld form. For $d=3$ and $d=4, I_{1}^{d=3}$ is the Einstein-Hilbert action which is equivalent to the Chern-Simons action and $I_{1}^{d=4}$ is the Einstein-Hilbert action which coincides with the Born-Infeld action upto the Euler density. In the case of $d=5$,

$I_{1}^{d=5}$ is the Einstein-Hilbert action, while $I_{2}^{d=5}$ leads to the Chern-Simons action. For $d=6$, $I_{1}^{d=6}$ is the Einstein-Hilbert action but $I_{2}^{d=6}$ is the Born-Infeld action.

The black hole solution to the action of Eq.(2.1) is given by [13,19]

$$
d s^{2}=-\left[1+\frac{r^{2}}{l^{2}}-\left(\frac{2 G_{k} M+\delta_{d-2 k, 1}}{r^{d-2 k-1}}\right)^{1 / k}\right] d t^{2}+\frac{d r^{2}}{1+\frac{r^{2}}{l^{2}}-\left(\frac{2 G_{k} M+\delta_{d-2 k, 1}}{r^{d-2 k-1}}\right)^{1 / k}}+r^{2} d \Omega_{d-2} .
$$

The mass of this black hole takes the form

$$
M\left(r_{+}\right)=\frac{r_{+}^{d-2 k-1}}{2 G_{k}}\left(1+\frac{r_{+}^{2}}{l^{2}}\right)^{k}-\frac{\delta_{d-2 k, 1}}{2 G_{k}}
$$




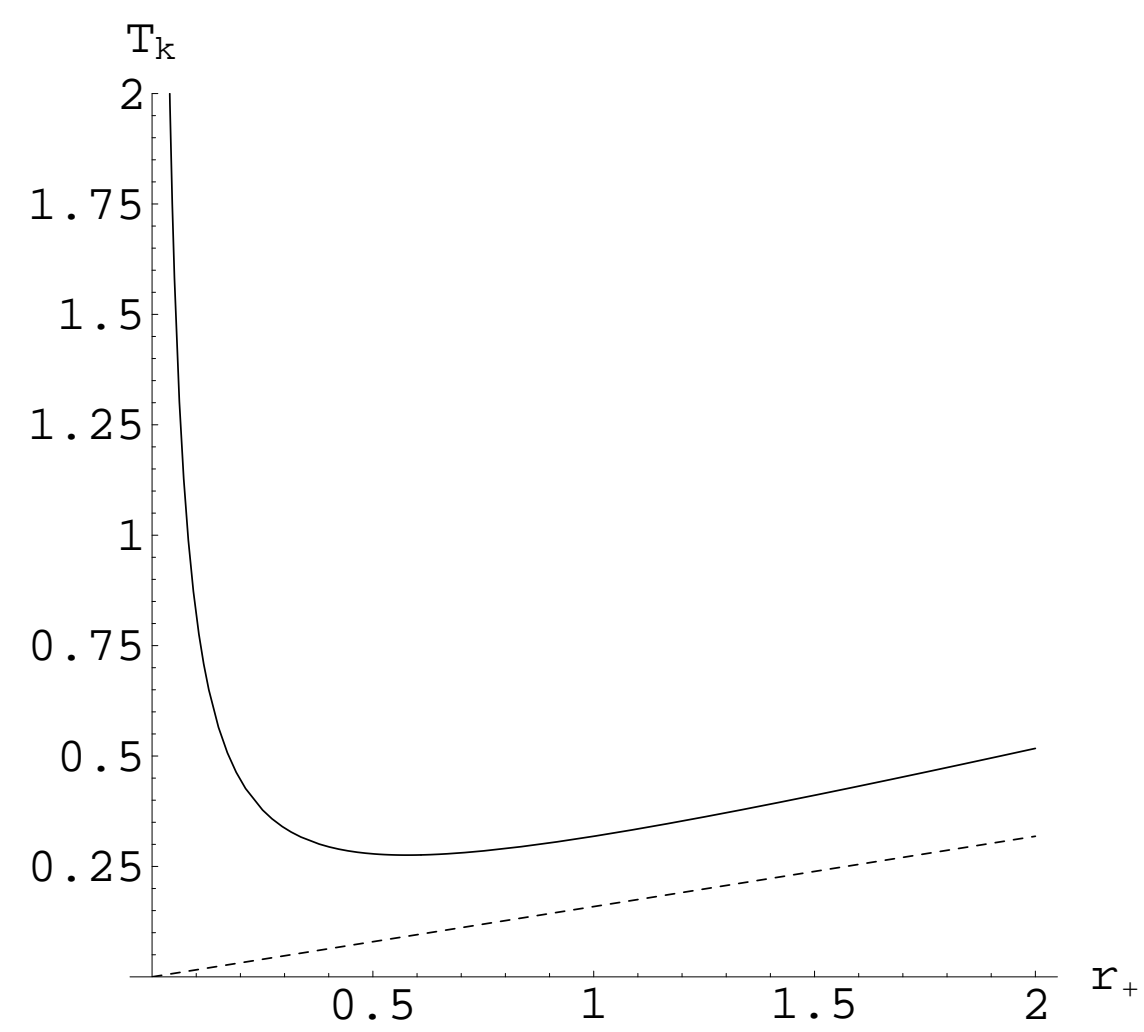

FIG. 1. Plot of the Hawking temperature as a function of the horizon radius $r_{+}$with $l=1$. For $d-2 k \neq 1$, we have the similar temperature behavior as in the case of the Schwarzschild-AdS black holes (solid line: $d=4, k=1$ ). In this case we have a minimum temperature of $T_{1}^{S A D S}=T_{c}=\sqrt{3} / 2 \pi$ at $r_{+}=r_{c}=1 / \sqrt{3}=0.58$. On the other hand, for $d-2 k=1$, we have the similar temperature behavior as in the case of the Chern-Simens black holes (dotted line: $d=3, k=1)$. Its absolute minimum is located at $T_{1}^{C S}=T_{c}=0\left(r_{+}=r_{c}=0\right)$, which corresponds to the MBTZ case.

which is a monotonically increasing function of the horizon radius $r_{+}$. The presence of the Kronecker delta function shows that there exist two different vacua $(M=0)$ with different causal structures.

In the case of $d-2 k \neq 1$, one finds the $d$-dimensional Schwarzschild-AdS black holes

$$
d s_{S A D S}^{2}=-\left[1+\frac{r^{2}}{l^{2}}-\left(\frac{2 G_{k} M}{r^{d-2 k-1}}\right)^{1 / k}\right] d t^{2}+\frac{d r^{2}}{1+\frac{r^{2}}{l^{2}}-\left(\frac{2 G_{k} M}{r^{d-2 k-1}}\right)^{1 / k}}+r^{2} d \Omega_{d-2}
$$

which possesses a continuous mass spectrum from $M\left(r_{+}\right)=\frac{r_{+}^{d-2 k-1}}{2 G_{k}}\left(1+\frac{r_{+}^{2}}{l^{2}}\right)^{k}$ to the thermal AdS with $M(0)=0: d s_{A D S}^{2}=-\left(1+r^{2} / l^{2}\right) d t^{2}+\left(1+r^{2} / l^{2}\right)^{-1} d r^{2}+r^{2} d \Omega_{d-2}^{2}$. Its Hawking temperature and heat capacity are given by [20]

$$
T_{k}^{S A D S}=\frac{1}{4 \pi k}\left[\frac{(d-1) r_{+}}{l^{2}}+\frac{d-2 k-1}{r_{+}}\right], \quad C_{k}^{S A D S}=\frac{2 \pi k r_{+}^{d-2 k}}{G_{k}}\left(\frac{r_{+}^{2}+r_{c}^{2}}{r_{+}^{2}-r_{c}^{2}}\right)\left(1+\frac{r_{+}^{2}}{l^{2}}\right)^{k-1}
$$

with the Boltzmann constant $k_{B}=1$ and the minimum length $r_{c}=l \sqrt{(d-2 k-1) /(d-1)}$. As is shown in Fig. 1, the Hawking temperature diverges at $r_{+}=0$. It has the minimum 


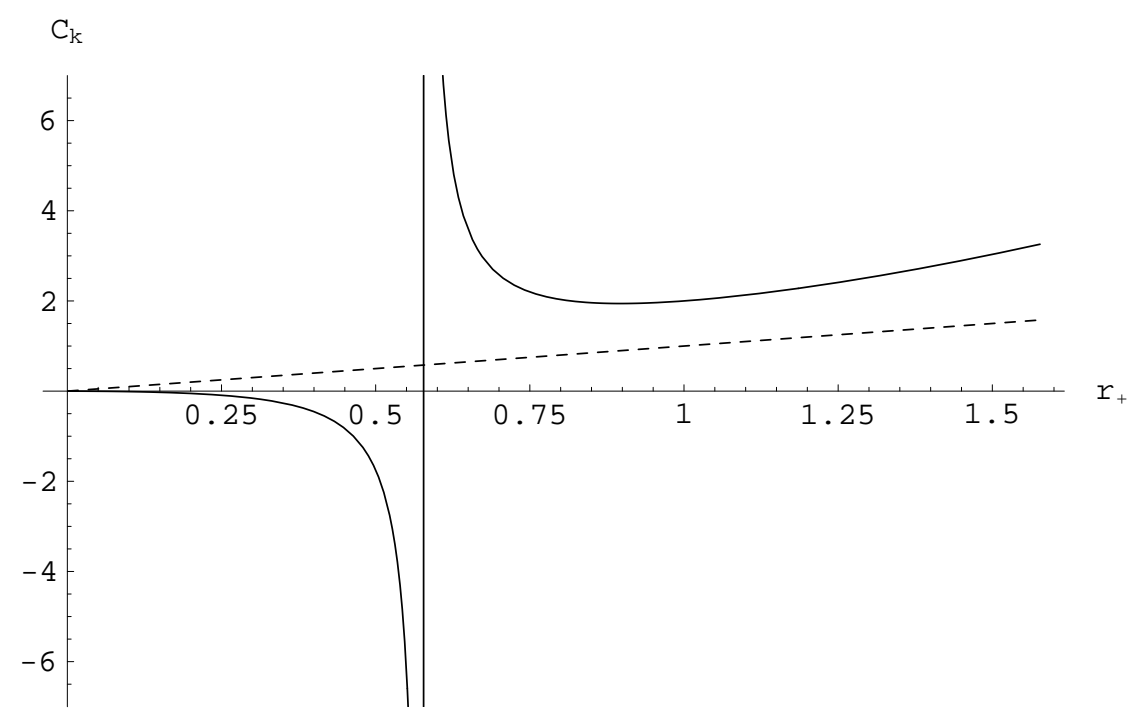

FIG. 2. Plot of the heat capacity as a function of $r_{+}$with $l=1$. For $d-2 k \neq 1$ (solid line: $d=4, k=1), C_{1}^{S A D S}$ has a pole at $r_{+}=r_{c}=0.58$. This shows that the phase transition at $T_{1}=T_{c}\left(r_{+}=r_{c}\right)$ is first-order. On the other hand, for $d-2 k=1($ dotted line: $d=3, k=1)$, the heat capacity is a monotonically continuous increasing function of $r_{+}$. We have a zero heat capacity $C_{1}^{C S}=0$ at $r_{+}=r_{c}=0$ which corresponds to the MBTZ case. It shows that the phase transition between NBTZ with $M \neq 0$ and MBTZ with $M=0$ is second-order.

value of the critical temperature $T_{c}=(d-1) r_{c} / 2 \pi k l^{2}$ at $r_{+}=r_{c}$, and grows linearly for large $r_{+}$. Actually the Hawking temperature for $k \neq 1$ and $d \geq 5$ has the similar behavior as in the $d=4$ Schwarzschild-AdS black hole. As is shown Fig. 2, the heat capacity has an unbounded discontinuity at $r_{+}=r_{c}$, signaling a phase transition from negative heat capacity to positive one. Roughly speaking, thermal behavior split into two branches. For $r_{+}<r_{c}$, the heat capacity is negative and thus the black hole state cannot be in equilibrium with the heat bath at temperature $T_{0}$. On the other hand, for $r_{+}>r_{c}$, the heat capacity is positive and thus the black hole state can be in equilibrium with the heat bath. If $r_{+}$ exceeds an unstable equilibrium position $\left(r_{u}\right)$ in the order of $r_{u}<r_{c}<r_{s}$ where $r_{s}$ is a locally stable position, the Schwarzschild-AdS black hole can reach equilibrium with a heat bath of temperature $T_{0}>T_{c}$. Here $T_{0}$ corresponds to two equilibrium states of radii $r_{u}$ and $r_{s}$ [13]. Explicitly, if $r_{+}>r_{u}$, the black hole evolves towards an equilibrium configuration at $r_{+}=r_{s}$. If either $T_{0}<T_{c}$ or $r_{+}<r_{u}$, the black hole continues to evaporate until its final stage. That is, it is unstable to decay into the thermal AdS space. This is a typical picture for the first-order Hawking-Page transition in even dimensions. It shows that the phase transition is sensitive to the initial black hole state $r_{+}$and the temperature $T_{0}$ of the heat bath. The higher order curvature-correction with $k \neq 1$ to the gravity never alters this feature of phase transition. In the case of $k=1$, one finds the area-law of the entropy: $S_{1}^{S A D S}=2 \pi r_{+}^{d-2} /(d-2) G_{1}=S_{B H}$. The entropy for $k=2$ case takes the form

$$
S_{2}^{S A D S}=\frac{4 \pi}{G_{k}}\left[\frac{r_{+}^{d-2}}{(d-2) l^{2}}+\frac{r_{+}^{d-4}}{d-4}\right]
$$

where one cannot find the area-law behavior because of the presence of the last term.

The other case is for odd dimensions with $d-2 k=1(k=n-1)$, called the Chern-Simons 
black holes as

$$
d s_{C S}^{2}=-\left[1+\frac{r^{2}}{l^{2}}-\left(2 G_{k} M+1\right)^{1 / k}\right] d t^{2}+\frac{d r^{2}}{1+\frac{r^{2}}{l^{2}}-\left(2 G_{k} M+1\right)^{1 / k}}+r^{2} d \Omega_{d-2}
$$

which possesses a continuous mass spectrum from $M\left(r_{+}\right)=\frac{r_{+}^{d-2 k-1}}{2 G_{k}}\left(1+\frac{r_{+}^{2}}{l^{2}}\right)^{k}-\frac{1}{2 G_{k}}$ to the massless AdS black holes $(M=0)$ with different topology: $d s_{M A D S}^{2}=-\left(r^{2} / l^{2}\right) d t^{2}+$ $\left(r^{2} / l^{2}\right)^{-1} d r^{2}+r^{2} d \Omega_{d-2}^{2}$. The temperature and heat capacity are given by, respectively,

$$
T_{k}^{C S}=\frac{1}{4 \pi k} \frac{(d-1) r_{+}}{l^{2}}, \quad C_{k}^{C S}=\frac{2 \pi k r_{+}}{G_{k}}\left(1+\frac{r_{+}^{2}}{l^{2}}\right)^{k-1} .
$$

In this case the Hawking temperature and heat capacity are not divergent at all. These both are monotonically increasing positive functions of $r_{+}$(see Fig. 1 and 2). The minimum temperature is given by $T_{k}^{C S}\left(r_{+}=0\right)=T_{c}=0$ for the MADS case. Roughly speaking, the presence of the negative cosmological constant $\Lambda$ makes it possible for the Schwarzschild-AdS black holes to reach the thermal equilibrium under the condition of $T_{0}>T_{c}$ and $r_{+}>r_{u}$. In the case of the Chern-Simons black holes, the heat capacity is always positive and therefore the equilibrium configuration is always reached, regardless of the initial black hole state $r_{+}$ and the temperature $T_{0}$ of the heat reservoir. The entropy is defined from the Euclidean path integral as

$$
S_{k}^{C S}=\frac{2 \pi k}{G_{k}} \int_{0}^{r_{+}}\left(1+\frac{r^{2}}{l^{2}}\right)^{k-1} d r .
$$

This is also a monotonically increasing functions of $r_{+}$. For $k=2$ case, we have

$$
S_{2}^{C S}=\frac{4 \pi r_{+}}{G_{k}}\left(1+\frac{r_{+}^{2}}{3 l^{2}}\right) .
$$

In case of the Schwarzschild-AdS black holes, there exists a first-order phase transition at $T_{k}^{S A D S}=T_{c}\left(r_{+}=r_{c}\right)$. The heat capacity $C_{k}^{S A D S}$ is positive for $r_{+}>r_{c}$, while it is negative for $r_{+}<r_{c}$. Also $C_{k}^{S A D S}$ has a simple pole at $r_{+}=r_{c}$. This confirms from the analysis of the free energy $\left(F=M-T_{H} S\right)$, although its exact transition point of $r_{+}=r_{c}=l$ for $k=1$ is different from $r_{+}=r_{c}=l / \sqrt{3}$ derived by the heat capacity. We note a sign change between

$$
F\left(r_{+} \rightarrow 0\right) \sim r_{+}^{d-2 k-1} / 2(d-2 k) G_{k} \text { and } F\left(r_{+} \rightarrow \infty\right) \sim-r_{+}^{d-1} / 2(d-2) G_{k} l^{2 k}
$$

which shows that a small black hole with $r_{+}<r_{c}$ is unstable to decay into thermal AdS space, while a large black hole with $r_{+}>r_{c}$ is stable.

However, we always have a positive heat capacity of $C_{k}^{C S}>0$ for the Chern-Simons black holes. Since the Chern-Simons black holes are exceptional class, there is no phase transition at all even though $F$ has a change in sign as is shown in Eq.(2.12). This disagreement comes from a difference between the canonical ensemble approach with fixed temperature and the free energy approach with variable temperature. Actually, there is no critical temperature $T_{c}$ which gives us a first-order phase transition in the Chern-Simons system. They are genuine 
gauge theories whose supersymmetric extension is known. These black holes can reach thermal equilibrium with a heat bath at any temperature. The positivity of heat capacity guarantees their stability under thermal fluctuations. If the heat capacity is negative, there is no stable thermal fluctuations [19]. Accordingly, a small Chern-Simons black hole with $r_{+}<l$ is stable against decay by the Hawking radiation. In three dimensions, this is known to be the non-rotating BTZ black hole (NBTZ). One recovers thermodynamic quantities for the NBTZ with $G_{1}=1 / 2$

$$
T_{1}^{C S} \rightarrow T_{H}=\frac{r_{+}}{2 \pi l^{2}}, \quad C_{1}^{C S} \rightarrow C_{J}=4 \pi r_{+}, \quad S_{1}^{C S} \rightarrow S_{B H}=4 \pi r_{+} .
$$

The NBTZ spectrum has a mass gap separating it from thermal AdS space. We are interested in constructing the Gott time machine to study the formation of the non-rotating BTZ black hole by a two-body collision process [21]. The NBTZ is defined by a hyperbolic isometry. We recall that the mass of NBTZ is $M>0$, while the point particle mass $m$ is related to $M$ by $m=2(1-\sqrt{-M})$. Then the point particle spectrum is given by $-1<M<0$ which belongs to a branch of conical deficits. Here $M=0$ corresponds to MBTZ and $M=-1$ is TADS. Let us introduce the static black hole with the same left and right generators. We choose $\rho_{L}=\rho_{R}=-T^{G}=\rho$ because isometries of the NBTZ are subject to identifications with $\left(\rho_{L}, \rho_{R}\right) \sim\left(-\rho_{L},-\rho_{R}\right)$. If the Gott condition is satisfied, $T^{G}$ is a hyperbolic generator and thus the Gott time machine results in formation of the NBTZ. The mass is given by an input parameter $p$ which depends on the initial data

$$
\frac{\operatorname{Tr} \rho}{2}=\cosh [\pi \sqrt{M}] \equiv p, \quad p \geq p_{*}=1
$$

which means that an order parameter for formation of NBTZ is the trace of the generator. This takes a critical value at the threshold for the black hole formation $\left(p=p_{*}\right)$. In other words, $p=p_{*}$ corresponds to the vacuum of the black hole (MBTZ), where the Gott generator becomes parabolic. From Eq.(2.14), one finds an exact formula for the formation of NBTZ in terms of the parameter $p$ as

$$
\pi \sqrt{M}=\cosh ^{-1}(p)=\ln \left[p+\sqrt{p^{2}-1}\right] .
$$

From the above, one determines the Choptuik scaling for formation of the NBTZ

$$
\sqrt{M}=\frac{r_{+}}{l}=\frac{\sqrt{2}}{\pi}\left(p-p_{*}\right)^{1 / 2},
$$

where implies that the Choptuik scaling exponent is given by $\gamma=1 / 2$.

If the Gott condition is not satisfied, one has an effective particle spacetime with an elliptic generator. In case of $-1<M<0$, a conical deficit angle $\alpha$ is introduced for an order parameter to describe the phase transition. Defining $p$ as

$$
\frac{\operatorname{Tr} \rho}{2}=\cos [\pi \sqrt{-M}] \equiv p, \quad p<p_{*}=1
$$

one has similarly the Choptuik scaling exponent with $\gamma=1 / 2$ as

$$
\alpha=2 \pi-2 \sqrt{2}\left(p_{*}-p\right)^{1 / 2} .
$$

In case of MBTZ with $M=0$, we have $p=p_{*}$ and $\alpha=2 \pi$. For TADS with $M=-1$, we have $\alpha=2 \pi-4>0$. 


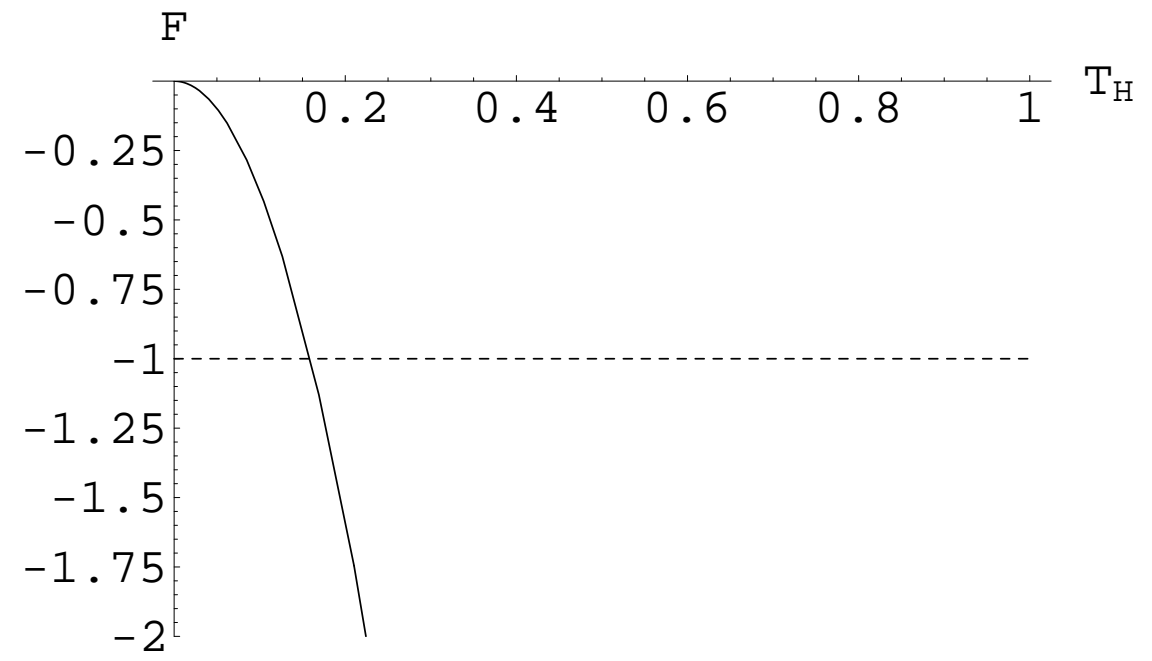

FIG. 3. Plot of the free energy as function of the Hawking temperature $T_{H}$ with $l=1$. The solid line represents the free energy behavior for the NBTZ case, while the dotted line denotes the constant negative free energy for the TADS case. Their values coincide with each other at the assumed critical temperature $T_{H}=T_{c}=1 / 2 \pi$.

\section{NO HAWKING-PAGE PHASE TRANSITION IN THREE DIMENSIONS}

In $d \geq 4$ case, Hawking-Page phase transition occurs between Schwarzschild-AdS black hole and thermal AdS space. This is possible because the presence of a negative cosmological constant is sufficient to render the canonical ensemble well defined for all Schwarzschild-AdS black holes. However, for Chern-Simons black holes (NBTZ case), the situation is quite different from those of Schwarzschild-AdS black holes. For our purpose, we express the free energy, energy and heat capacity in terms of the Hawking temperature as

$$
F_{N B T Z}=-E_{N B T Z}=-(2 \pi l)^{2} T_{H}^{2}, \quad C_{N B T Z}=S_{N B T Z}=2(2 \pi l)^{2} T_{H} .
$$

It is obvious that the NBTZ with $T_{H}=0$ leads to those for MBTZ case as

$$
F_{M B T Z}=E_{M B T Z}=C_{M B T Z}=S_{M B T Z}=0 .
$$

On the other hand, thermodynamic quantities for thermal AdS space are given by

$$
F_{T A D S}=E_{T A D S}=-1, \quad C_{T A D S}=S_{T A D S}=0
$$

with $T_{H}=0$.

At this stage, we introduce the assumed picture of the first-order Hawking-Page transition in three dimensions. A first-order phase transition may occur at $T_{H}=T_{c}=1 / 2 \pi l$ between NBTZ and TADS [22]. As is shown in Fig. 3, for $T_{H}<T_{c}$, the free energy of TADS is lower than that of NBTZ so that NBTZ is less probable than TADS. For $T_{H}>T_{c}$, the NBTZ is more probable than TADS. We raise the temperature of system gradually from the lower one. Then TADS dominates as long as $T_{H}<T_{c}$. When $T_{H}=T_{c}$, the TADS begins to be transformed into the NBTZ configuration. Also there exists discontinuity for energy and heat capacity between NBTZ and TADS. However, in three dimensions, one has a mass gap 


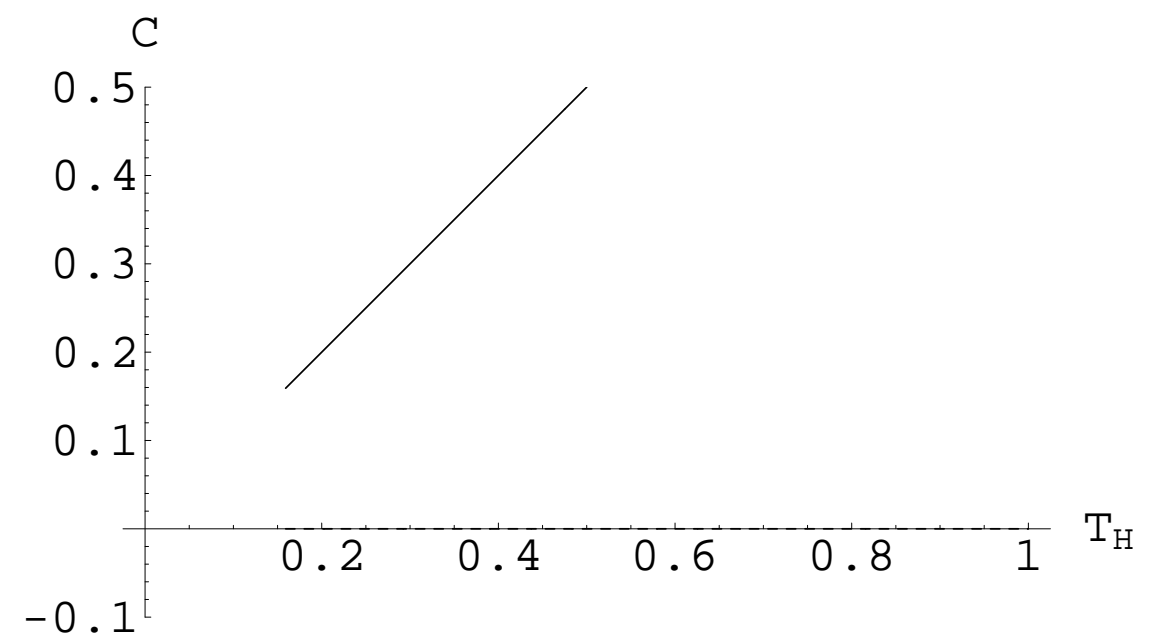

FIG. 4. Plot of the heat capacity as a function of the Hawking temperature with $l=1$. Below the assumed critical temperature $T_{H}=T_{c}=1 / 2 \pi$, the heat capacity for the TADS is zero. The heat capacity for NBTZ increases linearly for $T_{H}>T_{c}$. Here we have a finite jump of the heat capacity at $T_{H}=T_{c}$. However, even if the assumed transition occurs really, it does not imply the presence of the first-order Hawking-Page transition.

between MBTZ with $M=0$ and TADS with $M=-1$. A conical deficit interpreted as a point mass source appears between these. As was shown in the previous section, the branch of $-1<M<0$ is totally different from the branch of NBTZ with $M>0$. A second-order phase transition might be taken from MBTZ with $M=0$ to NBTZ with $M>0$ because the energy and heat capacity (entropy) are continuous [14]. This calculation is based on the semiclassical approximation for the black hole thermodynamics.

Alternatively, if one includes quantum fluctuations, there exits a possibility that the MBTZ is not the end of the Hawking evaporation and the end might be the TADS [23]. Then we may introduce the above assumed transition between NBTZ and TADS. It may correspond to a counterpart to the first-order Hawking-Page transition between SchwarzschildAdS black hole with $M>0$ and thermal AdS space with $M=0$. However, this phase transition turns out to be second-order because the heat capacity has not a pole but a finite jump at the assumed critical temperature $T_{H}=T_{c}$.

Consequently, the transitions between NBTZ and MBTZ (TADS) might be possible to occur in three dimensions but these do not belong to the first-order Hawking-Page transition.

\section{ACKNOWLEDGEMENT}

The author thanks to Gungwon Kang and Jungjai Lee for helpful discussions at the early stage of this work. This work was supported by the Korea Research Foundation Grant(KRF-2005-013-C00018). 


\section{REFERENCES}

[1] S. W. Hawking, Phys. Rev. D 14, 2460 (1976).

[2] G. 't Hooft, Nucl. Phys. B 335, 138 (1990).

[3] L. Susskind, hep-th/0204027.

[4] D. N. Page, hep-th/0409024.

[5] J. Maldacena, JHEP 0304, 021, (2003) 021 [hep-th/0106112].

[6] M. Banados, M. Henneaux, C.Teitelboim and J. Zanelli, Phys. Rev. D 48, 1506(1993)[grqc/9302012].

[7] S. Carlip, gr-qc/0503022.

[8] G. T. Horowitz and D. L. Welch, Phys. Rev. Lett. 71, 328(1993)[hep-th/9302126];

J. H. Horne and G.T. Horowitz, Nucl. Phys. B 368, 444(1992)[hep-th/9108001];

H. W. Lee and Y.S. Myung, Phys. Rev. D 58, 104013(1998) [hep-th/9804095];

H. W. Lee, N. J. Kim, and Y. S. Myung, Phys. Rev. D58, 084022(1998)[hepth/9803080]; Phys. Lett. B441, 83(1998)[hep-th/9803227].

[9] J. Maldacena, Adv. Theor. Math. Phys. 2, 231 (1998) [Int. J. Theor. Phys. 38, 1113 (1999)] [hep-th/9711200];

S. S. Gubser, I. R. Kelebanov and A. M. Polyakov, Phys. Lett. B428, 105 (1998)[hepth/9802109];

E. Witten, Adv. Theor. Math. Phys. 2, 253 (1998) [hep-th/9802150].

[10] S. W. Hawking, hep-th/0507171.

[11] S. W. Hawking and D. N. Page, Commun. Math. Phys. 87, 577 (1983).

[12] D. Birmingham, I. Sachs, and S. N. Solodukhin, Phys. Rev. D67, 104026(2003)[hepth/0212308].

J. Barbon and E. Rabinovici, JHEP 0311, 047(2003)[hep-th/0308063];

M. Kleban, M. Porrati and R. Rabadan, JHEP 0410, 030(2004)[hep-th/0407192];

S. Solodukhin, Phys. Rev. D 71, 064006 (2005)[hep-th/0501053];

J.Barbon and E. Rabinovici, Fortsch. Phys. 52, 642(2004)[hep-th/0403268]; hepth/0503144.

[13] J. Crisostomo, R. Troncoso, and J. Zanelli, Phys. Rev. D 62, 084013 (2000)[hepth/0003271].

[14] R. G. Cai, Z.-J. Lu, Y.-Z. Zhang, Phys. Rev. D 55, 853(1997)[gr-qc/9702032].

[15] R. -G. Cai and J.-H. Cho, Phys. Rev. D 60, 067502(1999)[hep-th/9803261].

[16] Y. S. Myung, Phys. Lett. B 579, 205(2004) [hep-th/0310176].

[17] Y. S. Myung and H. W. Lee, hep-th/0506031.

[18] J. Maldacena and A. Strominger, JHEP 9812, 005(1998)[hep-th/9804085].

[19] L. Alejandro Correa-Borbonet, hep-th/0506018.

[20] R.-G. Cai, Y. S. Myung, and N. Ohta, Class. Quant. Grav. 18, 5429 (2001)[hepth/0105070].

[21] D. Birmingham and S. Sen, Phys. Rev. Lett. 84, 1074 (2000)[hep-th/9908150].

[22] Y. Kurita and M. Sakami, hep-th/0403091.

[23] G. Lifschytz and M. Ortiz, Phys. Rev. D 49, 1929 (1994)[gr-qc/9310008]. 